\title{
Knowledge, Attitude, and Practice regarding Anaphylaxis among Pediatric Health Care Providers in a Teaching Hospital
}

\author{
Sibabratta Patnaik ${ }^{1}$ Samarth Krishna ${ }^{2}$ Mukesh Kumar Jain ${ }^{1}$ \\ ${ }^{1}$ Department of Pediatrics, Kalinga Institute of Medical Sciences, \\ Bhubaneswar, Odisha, India \\ 2 Kalinga Institute of Medical Sciences, Bhubaneswar, Odisha, India \\ Address for correspondence Sibabratta Patnaik, MBBS, MD, \\ Department of Pediatrics, Kalinga Institute of Medical Sciences, \\ Bhubaneswar 751024, Odisha, India \\ (e-mail: drsbpatnaik45@gmail.com).
}

J Child Sci 2020;10:e224-e229.

\begin{abstract}
Anaphylaxis is a severe, life threatening generalized or systemic hypersensitivity reaction which requires prompt, accurate diagnosis, and appropriate management. The objective of the study is to assess the extent of knowledge, management skills, and attitude on anaphylaxis among pediatric health care providers of a tertiary care teaching hospital. This was a cross-sectional study done in the department of pediatrics, which included faculty, residents, interns, and nurses. The knowledge, attitude, and practice were assessed using a standardized questionnaire comprised of 35 questions. The questionnaire was distributed and collected in person to all the participants. Informed consent was obtained from all participants and confidentiality was ensured at all stages. Collected data were analyzed using SPSS version 20 . The mean of total knowledge score (TKS) was $7.36 \pm 1.42$. TKS of faculty was $8.36 \pm 1.15$, in residents $7.81 \pm 1.25$, in interns $7.46 \pm 1.15$, and in nurses $6.69 \pm 1.57$. Knowledge scores of faculty, residents, and interns were significantly higher than that of nurses while there was no significant difference between faculty, residents, and interns. The majority of participants have positive attitude toward anaphylaxis. Incorrect practice is followed by most of the participants. The extent of knowledge about anaphylaxis

Keywords

- allergy

- epinephrine

- glucocorticoid

- TKS among pediatric health care providers is moderate but management skills remains suboptimal. Thus, proper training, through continued medical educations and programs to improve abilities to recognize and manage anaphylaxis are very crucial. Differential approach may be followed to different groups; nurses need extra training for optimal management of anaphylaxis.
\end{abstract}

\section{Introduction}

Anaphylaxis is a severe, life threatening generalized or systemic hypersensitivity reaction which is characterized by rapidly developing airway and/or breathing and/or circulation problems usually associated with skin and mucosal changes. ${ }^{1}$ Currently, more than $25 \%$ of total population of India is sensitized to different allergens. ${ }^{2}$ Recent studies

received

August 25, 2020

accepted after revision

October 6, 2020
DOI https://doi.org/

10.1055/s-0040-1720955. ISSN 2474-5871. show that the prevalence and incidence of allergic disorders have increased globally in the past 20 years. ${ }^{3}$ Many studies have demonstrated that the most common triggers are drugs in adults, and food in pediatric population. ${ }^{4}$

Epinephrine is the first line drug for the treatment of anaphylaxis and several studies conducted previously reveal that there is lack of knowledge regarding its dose and route of administration. There is confusion in selecting first-line
Copyright @ 2020 Georg Thieme Verlag License terms KG Stuttgart · New York

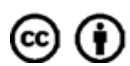


drugs for treating anaphylaxis in an emergency situation among health care professionals (HCPs). ${ }^{5-7}$ If severe anaphylaxis occurs, then the time frame for its management is quite limited. As anaphylaxis is a life-threatening condition and can happen at any time, the HCPs should always be prepared to deal with this condition, and knowledge about this dangerous disease should be kept up to date among HCPs.

The objective of this study was to assess the knowledge regarding recognition of symptoms, signs, and management of anaphylaxis among HCPs in a tertiary health care hospital and to assess the attitude and practices for the management of anaphylaxis among them.

\section{Materials and Methods}

This was a cross-sectional study among HCPs in a tertiary care teaching hospital. The department of pediatrics was surveyed for the study, including faculty, residents, interns, and nurses. The Institutional Ethics Committee of KIMS, Bhubaneswar, approved the study (KIIT/KIMS/IEC/29/2019).

The questionnaire was comprised of 35 questions pertaining to knowledge, practice, and attitude. The first six questions retrieved information regarding demographic data of health care providers (profile of HCP, age, gender, education, number of years in practice, and place of practice). The next 10 questions focused on knowledge regarding anaphylaxis including definition of anaphylaxis, common symptoms and causes, type of hypersensitivity reaction, first-line drug to use along with appropriate dose, and preferred route of administration. The next 13 questions appraised practice regarding management of anaphylaxis with questions on supportive treatment, site of administration, dose concentration, administration time of adrenaline, etc. The last six questions assessed attitudes of health care providers toward anaphylaxis. After preparing the questionnaire, it was sent to three experts (one Professor of Pediatrics with interest in rheumatology, one Associate Professor in Pediatrics with interest in asthma and allergy, and one pediatric intensivist) for validation and modification was done as per their suggestions. After that the questionnaire was pretested among five subjects.

The questionnaire was distributed and collected in person from all the participants. Informed consent was taken from all and confidentiality was ensured at all stages. The participants were divided into four groups: faculty, residents, interns, and nurses. The data analysis was performed using SPSS version 20. Correct and incorrect responses of each knowledge question were given 1 and 0 marks, respectively, with maximum attainable total knowledge score (TKS) of 10. Percentages, mean, standard deviation, ANOVA test were used for statistical analysis. $p$-Values less than 0.05 were considered as significant.

\section{Results}

The questionnaire was distributed to 130 health care providers and out of them 125 completed the questionnaire, with a response rate of $96.15 \%$. Out of the total 125 respon-
Table 1 Socio-demographics characteristics of participants

\begin{tabular}{|l|l|}
\hline Characteristics & \\
\hline Age (Mean \pm SD) in years & $28.19 \pm 8.785$ \\
\hline Gender $n(\%)$ & \\
\hline Female & $77(61.6)$ \\
\hline Male & $48(38.4)$ \\
\hline Profile $n(\%)$ & \\
\hline Faculty & $14(11.2 \%)$ \\
\hline Residents & $21(16.8 \%)$ \\
\hline Nurses & $42(33.6 \%)$ \\
\hline Interns & $48(38.4 \%)$ \\
\hline
\end{tabular}

Abbreviation: SD, standard deviation.

dents, $61.6 \%$ were females and $38.4 \%$ males. Among them, $11.2 \%$ were faculty, $16.8 \%$ residents, while $33.6 \%$ were nurses, and $38.4 \%$ interns (-Table $\mathbf{1}$ ).

\section{Knowledge regarding Anaphylaxis}

Among all surveyed, $65.6 \%$ chose severe allergic reaction as best definition of anaphylaxis. When they were specifically asked about the type of hypersensitivity reaction of anaphylaxis $97.6 \%$ respondents answered type I but $2.4 \%$ answered type IV. When asked about first line of treatment of anaphylaxis out of 125 responses 119 (95.2\%) respondents answered epinephrine, while three $(2.4 \%)$ respondents chose glucocorticoid. Only 88 (70.4\%) respondents decided to give epinephrine intramuscularly, while $27(22.3 \%)$ respondents chose intravenous as the route of administration. Twenty-eight (22.4\%) participants were able to tell the correct dose of epinephrine (-Table 2).

The mean of TKS was $7.36 \pm 1.42$. TKS of faculty was $8.36 \pm 1.15$, while in residents $7.81 \pm 1.25$, in interns $7.46 \pm 1.15$, and in nurses $6.69 \pm 1.57$. Knowledge score of faculty, residents, and interns was significantly higher than that of nurses while there were no significant differences between faculty, residents, and interns (-Table 3 ).

\section{Attitude regarding Ability to Treat Anaphylaxis}

Among the respondents only 56\% were confident in their ability to treat patients with anaphylaxis. $34.4 \%$ of the respondents would prefer to refer the patient to an allergist. A majority of respondents would advise their patients to get allergy testing done, to keep away from known allergens, while a few would prescribe antihistaminic. $88.8 \%$ of HCPs would advise their patients for follow-up with them. High proportions of health care providers (99.2\%) were willing to participate in training sessions for the management of anaphylaxis, while all the respondents (100\%) were supportive to the idea of promoting public awareness regarding anaphylaxis (-Table 2 ).

\section{Practice regarding Management of Anaphylaxis}

Among all the respondents, $56.8 \%$ had seen a case of anaphylaxis, however, only 38 (30.4\%) out of them had managed a 
Table 2 Detailed questionnaire

\begin{tabular}{|c|c|c|}
\hline Questions & Correct & Incorrect \\
\hline Knowledge & $n(x)$ & $n(x)$ \\
\hline 1. Best definition of anaphylaxis & $82(65.6)$ & $43(34.4)$ \\
\hline 2. What type of hypersensitivity reaction is anaphylaxis? & $122(97.6)$ & $3(2.4)$ \\
\hline 3. What is the first line of drug for anaphylaxis? & $119(95.2)$ & $6(4.8)$ \\
\hline 4. What is the second line of drug for anaphylaxis? & $75(60)$ & $50(40)$ \\
\hline 5. What is the dose of the drug (of choice)? & $28(22.4)$ & $97(77.6)$ \\
\hline 6. The preferred route for administration of the most important drug in anaphylaxis? & $88(70.4)$ & $37(29.6)$ \\
\hline 7. Write common symptoms of anaphylaxis. & $106(84.8)$ & $19(15.2)$ \\
\hline 8. Foods most likely to cause severe allergy? & $116(92.8)$ & $9(7.2)$ \\
\hline 9. Name two common medications likely to cause severe allergy. & $107(85.6)$ & $18(14.4)$ \\
\hline 10. What will be the symptom after re-exposure to the same agent? & $77(61.6)$ & $48(38.4)$ \\
\hline Attitude & Positive & Negative \\
\hline 11. Are you confident in your ability to treat patient with anaphylaxis? & $70(56.0)$ & $55(44)$ \\
\hline 12. How often do you refer your patients with suspected allergy? & $43(34.4)$ & $82(65.6)$ \\
\hline 13. Any advice to patients to prevent/minimize future reaction? & $115(92)$ & $10(8)$ \\
\hline 14. Would you like to advice the patient for follow-up? & $111(88.8)$ & $14(11.2)$ \\
\hline 15. Would you like to participate in training sessions on anaphylaxis? & $124(99.2)$ & $1(0.8)$ \\
\hline 16. Would you support the idea of promoting public awareness regarding anaphylaxis? & $125(100)$ & $0(0)$ \\
\hline Practice & Correct & Incorrect \\
\hline 17. Have you seen a case of anaphylaxis? & $71(56.8)$ & $54(43.2)$ \\
\hline 18. Have you managed a case of anaphylaxis? & $38(30.4)$ & $87(69.6)$ \\
\hline 19. How do you manage a case of anaphylaxis, i.e., supportive treatment other than adrenaline? & $21(55.3)$ & $17(44.7)$ \\
\hline 20. In which site do you administer adrenaline? & $9(23.7)$ & $29(66.3)$ \\
\hline 21. What concentration of adrenaline do you use for IM route? & $13(34.2)$ & $25(65.8)$ \\
\hline 22. What concentration of adrenaline do you use for IV route? & $23(60.5)$ & $15(39.5)$ \\
\hline 23. What is the dose of adrenaline you administer? & $16(42.1)$ & $22(57.9)$ \\
\hline 24. How do you manage patients, reporting previous anaphylaxis? & $17(44.7)$ & $21(55.3)$ \\
\hline 25. Which antihistaminic you prefer in the treatment of anaphylaxis? & $30(78.9)$ & $8(21.1)$ \\
\hline 26. When does the effect of IV hydrocortisone begin? & $17(44.7)$ & $21(55.3)$ \\
\hline 27. Administration time of adrenaline? & $32(84.2)$ & $6(15.8)$ \\
\hline 28. Is there any replacement of adrenaline? & $20(52.6)$ & $18(47.4)$ \\
\hline 29. What is the strength of adrenaline available in your hospital? & $20(52.6)$ & $18(47.4)$ \\
\hline
\end{tabular}

Abbreviations: IM, intramuscular; IV, intravenous.

Table 3 Total knowledge score (TKS)

\begin{tabular}{|l|l|l|l|l|l|}
\hline TKS $(M \pm S D)$ & Faculty $(M \pm S D)$ & Residents $(M \pm S D)$ & Interns $(M \pm S D)$ & Nurses (M \pm SD) & Significance \\
\hline $7.36 \pm 1.42$ & $8.36 \pm 1.15$ & $7.81 \pm 1.25$ & $7.46 \pm 1.15$ & $6.69 \pm 1.57$ & 0.000 \\
\hline
\end{tabular}

Abbreviations: M, mean; SD, standard deviation; TKS, total knowledge score.

Note: Faculty and Nurse: $p$-value 0.000 (significant); faculty vs. residents: 1 ; faculty vs. interns: 0.162 ; intern vs. nurse: 0.041 (significant); intern vs. residents: 1 ; residents vs. nurse: 0.012 (significant).

case of anaphylaxis. When asked about symptoms of anaphylaxis, breathing difficulty, itching, hypotension, and rashes were the most common answers. Egg, milk, prawns, and peanut were answered as the most common allergens encountered in their practice. The answers for the most common medications that they had seen which causes anaphylaxis were penicillin, sulfonamides, cephalosporins, and nonsteroidal-anti-inflammatory agents. 
Out of 38 who had personally managed anaphylaxis, only $23.5 \%$ respondents chose anterolateral thigh as the preferred site of administration of epinephrine. Only $34.1 \%$ of the respondents could correctly answer 1:1,000 as the concentration of epinephrine for intramuscular administration. When asked about the administration time of epinephrine 32 (84.2\%) out of the 38 respondents preferred to administer epinephrine immediately. The respondents were confused when asked about when the effect of IV hydrocortisone began. $44.7 \%$ respondents answered, "in 3 to 4 hours" while $55.3 \%$ of 38 respondents chose "immediately." When asked about the strength of epinephrine available in hospital which they had used, only 52.6\% were able to answer $1: 1,000$. While asked about any replacement of epinephrine for use in anaphylaxis, $47.3 \%$ believed that there is replacement of epinephrine whereas $52.7 \%$ believed that there is no replacement of epinephrine (-Table 2 ).

\section{Discussion}

Anaphylaxis is a serious allergic reaction that demands immediate attention as it is rapid in onset and may result in fatal outcome. The main objective of this study is to assess the extent of knowledge, attitude, and practice among consultants, residents, nurses, and interns in a pediatric department.

Droste and Narayan demonstrated that the knowledge deficit regarding anaphylaxis, among junior or senior doctors in most medical and surgical specialties is equally common. ${ }^{7}$ Many studies have proven that if there is failure or delay in administering the recommended first-line treatment, epinephrine, the mortality risk increases. $2,3,5$

The incidence of anaphylaxis is approximately 50 to 2,000 episodes per 100,000 persons and has a lifetime prevalence of 0.05 to $2.0 \%{ }^{8}$ The dose and route of administration of epinephrine for the management of cardiac arrest is different from that used in anaphylaxis. Previous reports have highlight- ed the confusion among many early-career doctors in the emergency department regarding the administration of epinephrine. ${ }^{6}$ Many reports demonstrated that the incorrect administration of epinephrine to patients with true or suspected anaphylaxis treated in hospital led to various complications including cardiac complications, stroke, and death. ${ }^{7,9,10}$ Intramuscular epinephrine is the treatment of choice. The dose is $0.01 \mathrm{mg} / \mathrm{kg}$ with a maximum dose of $0.5 \mathrm{mg}$ and it can be repeated every 5 to 15 minutes as necessary. In anaphylaxis, epinephrine should be administered as soon as possible. ${ }^{11}$ Antihistaminic can control urticaria and pruritus, but is not the treatment of choice as it does not help in controlling systemic manifestations. Corticosteroids should be considered as second-line drugs, as they might prevent biphasic reaction, but their effect usually starts after few hours. ${ }^{3}$ Though epinephrine is recommended by various published guidelines, the frequency of its use in cases of acute anaphylaxis is still suboptimal. 3,4,12,13 The overall knowledge regarding the use of epinephrine has been improving after World Allergy Organization published position paper on epinephrine for anaphylaxis in 2008. ${ }^{14}$ Clinical criteria for the diagnosis of anaphylaxis as per guidelines from the European Academy of Allergy and Clinical Immunology are provided in a separate table. This definition of anaphylaxis was followed in the present study ${ }^{11,13}$ ( - Table 4).

Thus, ameliorating health care providers' knowledge and practice to accurately diagnose and treat anaphylaxis is pivotal. The behavior and attitude of the professional facing a case of anaphylaxis is as crucial as that person's knowledge and management.

In regard to knowledge of anaphylaxis, $65 \%$ of respondents chose the accepted definition of anaphylaxis. This figure is comparatively less than that obtained from a survey of pediatric emergency department staff in Singapore. ${ }^{15}$ A survey from Bagalkot, India has shown that $90.1 \%$ of physicians prescribe epinephrine as the first-line medicine; with $38.3 \%$ preferring

Table 4 Clinical criteria for diagnosing anaphylaxis (based on Refs. ${ }^{11}$ and ${ }^{13}$ )

\begin{tabular}{|l|}
\hline Anaphylaxis is highly likely when any one of the following three criteria is fulfilled: \\
\hline $\begin{array}{l}\text { 1. Acute onset of an illness (minutes to several hours) with the involvement of the skin, mucosal tissue, or both (e.g., generalized } \\
\text { hives, pruritus or flushing, swollen lips-tongue-uvula and at least one of the following. }\end{array}$ \\
\hline a. Respiratory compromise (e.g., dyspnea, wheeze-bronchospasm, stridor, reduced PEF, hypoxemia). \\
\hline b. Reduced BP or associated symptoms of end-organ dysfunction (e.g., hypotonia [collapse], syncope, incontinence). \\
\hline 2. Two or more of the following that occur rapidly after exposure to a likely allergen for that patient (minutes to several hours): \\
\hline a. Involvement of the skin-mucosal tissue (e.g., generalized hives, itch-flush, swollen lips-tongue-uvula. \\
\hline b. Respiratory compromise (e.g., dyspnea, wheeze-bronchospasm, stridor, reduced PEF, hypoxemia). \\
\hline c. Reduced BP or associated symptoms (e.g., hypotonia [collapse], syncope, incontinence). \\
\hline d. Persistent gastrointestinal symptoms (e.g., crampy abdominal pain, vomiting). \\
\hline 3. Reduced BP after exposure to known allergen for that patient (minutes to several hours): \\
\hline a. Infants and children: low systolic BP (age specific) or $>30 \%$ decrease in systolic BP. ${ }^{\text {a }}$ \\
\hline b. Adults: systolic BP of <90 mm Hg or $>30 \%$ decrease from that person's baseline. \\
\hline
\end{tabular}


intramuscular route while $51.7 \%$ use intravenous epinephrine. ${ }^{16}$ Our study shows that $95.2 \%$ of respondents preferred epinephrine as first-line drug and 70.4\% respondents preferred the intramuscular route of administration. The standard pediatric dose is $0.01 \mathrm{mg} / \mathrm{kg}$ but $77.6 \%$ of the respondents were not able to answer the correct dose with only $22.4 \%$ of the respondents answering correctly. This response rate is comparable to the response rate reported in a previous study conducted in Mandya, Karnataka (20.34\%). ${ }^{17}$ In a recent study from Iran, it was found that the knowledge level of general practitioners and pediatricians on the management of anaphylaxis due to food allergy is suboptimal. The investigators found that $71.4 \%$ preferred subcutaneous epinephrine while $21 \%$ did not believe in using epinephrine for anaphylaxis. ${ }^{18}$ We found that in comparison to nurses, the knowledge level was significantly higher in faculty physicians, residents, and interns $(p=0.000,0.012$, and 0.041, respectively). Baççıŏlu and Yılmazel Uçar had shown in his paper, that the physicians had significantly higher knowledge than nurses in managing anaphylaxis. ${ }^{5}$ No significant difference was found between faculty, residents, and interns (-Table 3). The higher knowledge score in interns may be due to the fact that they had recently taken the final MBBS examination and thus had good theoretical knowledge, despite their limited clinical experience.

Our study revealed that $88.8 \%$ of HCPs would advise their patients to return for follow-up. Follow-up of a patient after an episode of anaphylaxis is a must to prevent mortality and morbidity because of biphasic reactions. So, observing the patient for at least 2 to 4 hours after the administration of epinephrine/other adjunctive therapies is critical to watch for and treat potential biphasic reaction. However, follow-up of the patient in the outpatient setting with an Allergy specialist preferably (but also with their primary care that may choose to refer to an allergy specialist) is important for facilitating workup of potential triggers for the anaphylactic reaction. In addition, patients who experience anaphylaxis and come under medical care should be prescribed an epinephrine autoinjector if at all possible. ${ }^{19}$ A study conducted in Kuwait revealed that $72.7 \%$ of respondents preferred to refer patients to an allergist, while in our study $34.4 \%$ respondents would prefer to refer patients to an allergist. ${ }^{20}$ Referral of a patient to an allergist is appropriate, as they help in identifying the allergen and establish a long-term management plan. In a survey from Miami, more than $70 \%$ of pediatric emergency medicine physicians referred their patients to allergists. ${ }^{21} \mathrm{We}$ observed that only $56 \%$ of the respondents felt confident that they could treat a case of anaphylaxis which was lower compared with a study conducted in Hyderabad among general practitioners and homeopaths, where $77.5 \%$ of subjects were confident that they could appropriately manage a case of anaphylaxis. $^{22}$ This might be due to inclusion of early career physicians, namely interns as well as nurses in our study.

In regard to practice and management skills assessment, our study showed only $56.8 \%$ of respondents had seen a case of anaphylaxis and only $30.4 \%$ of the respondents had managed a case of anaphylaxis. By contrast, a study conducted in Singapore revealed $89.4 \%$ of HCPs had seen a case of anaphylaxis. ${ }^{15}$ Our study demonstrates that a significant percentage of respondents did not know the correct dosage of epinephrine (57.9\%) and site of administration (66.3\%). However, this response rate appears to be comparable with responses rates to similar questions in surveys administered in several other countries. For example, a study conducted in United Kingdom observed that $34.1 \%$ of the respondents answered 1:1,000 as the recommended dose concentration of epinephrine. ${ }^{7}$ Only $52.6 \%$ of South Korean health care providers, who had managed anaphylaxis, could correctly tell the strength of adrenaline available in hospital. ${ }^{23}$

Regarding the onset of action of adjunctive therapies, including intravenous corticosteroids, in our study, 55.3\% of the respondents answered immediate action of IV hydrocortisone whereas $42.7 \%$ responded with the correct response of within 3 to 4 hours.

Similarly, in a previous study $55.4 \%$ of subjects responded that action of IV hydrocortisone starts immediately while $34.4 \%$ responded correctly that the onset of action for this medication was 3 to 4 hours. ${ }^{15}$ Many previous studies reveal that the majority of health care providers administer epinephrine immediately, which is similar to our findings. $47.4 \%$ of our respondents answered that there are substitute medications for epinephrine, although data on a comparable replacement for epinephrine is not available. Most of the participants are interested to attend training programs on anaphylaxis and all were supportive of the need to promote public awareness regarding anaphylaxis.

\section{Conclusion}

Our study has the limitation of being a single-center study with a small sample size, in which only $30.4 \%$ of surveyed HCPs had managed a case of anaphylaxis. However, the study shows that the level of knowledge regarding anaphylaxis is fair among both experienced and early career physicians but suboptimal among nurses. Though HCPs express confidence in their ability to manage anaphylaxis, practical management of anaphylaxis needs to be improved in all HCPs. Therefore, proper training, continued medical educations, and programs to bridge the knowledge gap and improve abilities to recognize and manage anaphylaxis remain very important. Differential approach in the form of model patients, simulated patients, extra training from allergy/ immunology specialists, etc., should be applied to improve the knowledge, attitude, and practice among HCPs.

\section{Acknowledgment}

The authors are thankful to Indian Council of Medical Research, New Delhi for considering this as a Short Term Studentship Project.

\section{References}

1 Altman AM, Camargo CAR Jr, Simons FE, et al. Anaphylaxis in America: a national physician survey. J Allergy Clin Immunol 2015;135(03):830-833

2 Shaikh WA, Shaikh SW. Allergies in India: an analysis of 3389 patients attending an allergy clinic in Mumbai, India. J Indian Med Assoc 2008;106(04):220-222, 222, 224 passim 
3 Simons FE. Anaphylaxis: recent advances in assessment and treatment.J Allergy Clin Immunol 2009;124(04):625-636, quiz 637-638

4 Simons FE, Ardusso LR, Dimov V, et al; World Allergy Organization. World Allergy Organization Anaphylaxis Guidelines: 2013 update of the evidence base. Int Arch Allergy Immunol 2013;162 (03):193-204

5 Baççıŏglu A, Yılmazel Uçar E. Level of knowledge about anaphylaxis among health care providers. Tuberk Toraks 2013;61(02): 140-146

6 Jose R, Clesham GJ. Survey of the use of epinephrine (adrenaline) for anaphylaxis by junior hospital doctors. Postgrad Med J 2007; 83(983):610-611

7 Droste J, Narayan N. Hospital doctors' knowledge of adrenaline administration in adults is deficient. Resuscitation 2010;81:1057-1058

8 Lieberman P, Camargo CA Jr, Bohlke K, et al. Epidemiology of anaphylaxis: findings of the American College of Allergy, Asthma and Immunology Epidemiology of Anaphylaxis Working Group. Ann Allergy Asthma Immunol 2006;97(05):596-602

9 Pumphrey RSH. Lessons for management of anaphylaxis from a study of fatal reactions. Clin Exp Allergy 2000;30(08):1144-1150

10 Manivannan V, Li JT, Prasad A, Campbell RL. Apical ballooning syndrome after administration of intravenous epinephrine during an anaphylactic reaction. Mayo Clin Proc 2009;84(09):845-846

11 Sampson HA, Wang J, Sicherer SH. Anaphylaxis. In: Kliegman RM, ed. Nelson Textbook of Pediatrics. 21st ed. Vol. 1;Philadelphia: Elsevier; 2020:1228-1234

12 O'Leary FM, Hokin B, Enright K, Campbell DE. Treatment of a simulated child with anaphylaxis: an in situ two-arm study. J Paediatr Child Health 2013;49(07):541-547

13 Muraro A, Roberts G, Worm M, et al; EAACI Food Allergy and Anaphylaxis Guidelines Group. Anaphylaxis: guidelines from the European Academy of Allergy and Clinical Immunology. Allergy 2014;69(08):1026-1045
14 Kemp SF, Lockey RF, Simons FEWorld Allergy Organization ad hoc Committee on Epinephrine in Anaphylaxis. Epinephrine: the drug of choice for anaphylaxis. A statement of the World Allergy Organization. Allergy 2008;63(08):1061-1070

15 Ibrahim I, Chew BL, Zaw WW, Van Bever HP. Knowledge of anaphylaxis among Emergency Department staff. Asia Pac Allergy 2014;4(03):164-171

16 Prabhu SB, Yasmeen M. Treatment of anaphylaxis in adults: a questionnaire survey at S. Nijalingappa medical college hospital, Bagalkot, India. J Clin Diagn Res 2010;4:3474-3479

17 Drupad HS, Nagabushan H. Level of knowledge about anaphylaxis and its management among health care providers. Indian J Crit Care Med 2015;19(07):412-415

18 Gharagozlou M, Afrough R, Malekzadeh I, Tavakol M. Knowledge, attitude and practice of general practitioners and pediatricians regarding food allergy in Iran. Rev Fr Allergol 2019;59(06): 421-426

19 Ben-Shoshan M, Clarke AE. Anaphylaxis: past, present and future. Allergy 2011;66(01):1-14

20 Rushood MAI. Anaphylaxis management: knowledge and practice among pediatricians in Kuwait. Curr Pediatr Res 2017;21(02): 242-247

21 Grossman SL, Baumann BM, Garcia Peña BM, Linares MY, Greenberg B, Hernandez-Trujillo VP. Anaphylaxis knowledge and practice preferences of pediatric emergency medicine physicians: a national survey. J Pediatr 2013;163(03):841-846

22 Kumar PU, Jain P, Siruguri V, Surekha MV, Rao VV. Knowledge, attitude and practices of general practitioners and homeopaths regarding food allergy and anaphylaxis in India-a comparitive study. EJMR 2017;4(1):91-96. Doi: 10.24041/ejmr2017.40

23 Seo DH, Ye YM, Kim SC, et al. A single hospital survey of anaphylaxis awareness among health care providers and medical students. Allergy Asthma Respir Dis 2016;4(02):133-139 